\title{
Single-phase media hydrodynamics and heat transfer in heat exchangers with twisted profile tubes
}

\author{
Yu. M. Brodov, K. E. Aronson, A. Yu. Ryabchikov \\ \& M. A. Nirenstein \\ Turbine and Engines Department, Ural Federal University, Russia
}

\begin{abstract}
A profiled heat exchanger tube is the one in which some features have been incorporated into the tube geometry for heat transfer enhancement. They offer a perspective method of steam turbine shell-and-tube heat exchangers improvement. Twisted profile tubes (TPT) are widely used in power engineering. This paper presents some results of experimental and theoretical research of hydrodynamics and heat transfer in TPTs. It is revealed that the heat transfer coefficient for water flow in a TPT increases up to $80 \%$ compared to that of a plain tube. With a rise of media Reynolds number, the heat transfer rate in a TPT decreases in comparison to that of a plain tube, but for air flow in a TPT the heat transfer coefficients ratio does not depend on the Reynolds number value. Water flow hydraulic losses in TPTs increase from 15 to $100 \%$ depending on the tube profile parameters.
\end{abstract}

Keywords: hydrodynamics, heat transfer, twisted profile tubes, heat exchanger, enhancement.

\section{Introduction}

Twisted profile tubes' (TPTs) application to energy system heat exchangers is a quite technologically advanced and cost-effective way to improve their efficiency. TPTs have quite satisfactory reliability characteristics. Their bending stiffness which affects tube system oscillation characteristics in heat exchangers (and thus their reliability) is 15 to $20 \%$ below that of the plain tubes of which TPTs are made. In TPT tube systems both oscillation amplitude and stress values (in tube and tube sheet junctions) are lower than in the plain tube apparatuses. So in terms of the TPTs vibration characteristics higher reliability can be achieved 
by a proper tube partition design in TPT apparatuses according to Ryabchikov et al. [1].

The lower corrosion resistance of TPTs in comparison to the plain tubes depends on the tube material and TPT geometric characteristics (Aronson et al [2]). For non-ferrous metals the TPTs corrosion rate increases against that of the plain tubes by as much as $15 \%$. For austenitic stainless steel the rates of corrosion are similar. So TPTs reliability characteristics are well studied and this makes their application for energy heat exchangers both justified and reasonable.

Twisted profile tubes are usually made of plain tubes by running them through the planetary rollers. In this case helical grooves are formed on the tube outer surface and corresponding protrusions are formed on the inner one (see fig. 1). The application of the TPTs with a 3-start knurling is regarded to be the most expedient for energy heat exchangers because of the following considerations:

- good manufacturability compared, for example, to TPTs with a single-start groove;

- acceptable hydraulic resistance increase in TPT heat exchangers;

- acceptable reliability in terms of corrosion and vibration resistance.

For theoretical and experimental studies and descriptions of tube geometric characteristics the outer contour of the TPT cross-section is considered to be described as a quirked epicycloid. Experimental studies of geometric characteristics for more than 60 samples of TPTs have shown that in energy heat exchangers calculations and design it is necessary to take into account that:

1. surface area values for the TPT and the plain tube are essentially identical (the difference is less than 2.5\%);

2. TPT clear area is from 3 to $13 \%$ smaller than that of the plain tube, depending on profile parameters; the equivalent diameter of the TPT clear area is from 2 to $8 \%$ smaller than that of the plain tube;

3. TPT circumscribed circle diameter can exceed the outer diameter of the plain tube from 1 to $4 \%$, with the TPT knurling pitch being $s \geq 0.75 d_{\text {out }}$ (this is to be taken into consideration when assembling or manufacturing the heat exchanger).
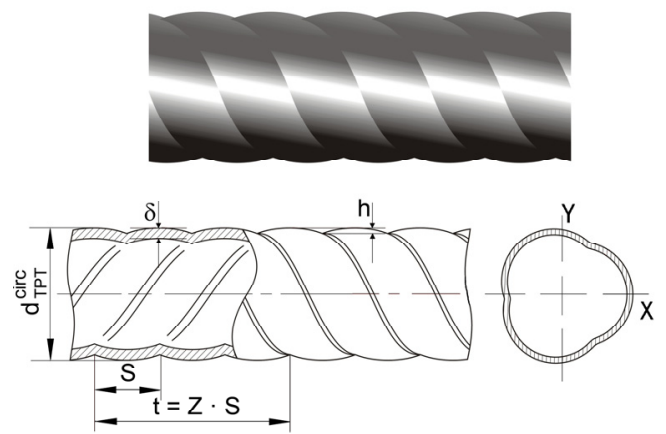

Figure 1: $\quad$ Twisted profile tube. $(h-$ groove depth; $s-$ groove pitch; $z-$ number of starts; $\delta$ - tube wall thickness; $d_{\text {TPT }}^{\text {circ }}-$ circumferential diameter.) 


\section{Heat exchange simulation for single-phase flow in TPTs}

Analytical research of hydrodynamics and heat exchange in tubes and channels with an artificial roughness is practically impossible because of the excessive complexity of the flow structure. This is adequately related to the twisted profile tubes, where an interaction occurs of axial, swirling and separated flows. The intensities of each of these flows, whose borders are practically impossible to locate because of their mutual imposition, are governed by the TPT profile parameters and by the axial flow regime. The flow is three-dimensional near the tube wall and its velocity consists of three components: one along the profile line (this component causes friction between the tube wall and the flow), one perpendicular to profile protrusions (this component causes mechanical energy losses in forming vortices) and the radial one. The values of these components and consequently the shares of friction and form losses could be estimated only by simulation. In close proximity to the protrusions the flow is of cellular pattern due to the horseshoe eddies formed there whose dynamics is affected by the form and size of the protrusion, while the vortices of various scales fill the flow area above the protrusion. Under such conditions certain simplification is required to construct an analytical flow model. On the basis of Prandtl-Taylor modified analogy a semi-empiric model was developed of heat transfer for single-phase flow. The essence of this analogy consists in summation of thermal resistances of different layers through which the heat flux passes (Reynolds [3]). For two-layer Prandtl-Taylor model the complete thermal resistance is a sum of thermal resistances of turbulent $R_{t}$ and molecular $R_{m}$ transfer:

$$
R=R_{t}+R_{m} \text {. }
$$

We also suppose that viscous sublayer thickness on a "smooth" area between the profile protrusions is substantially less than the height of the protrusion $h$. If it is so, then the wall zone thermal resistance $R_{m}$ entirely depends on the flow hydrodynamics between the protrusions, and the mode of the "developed roughness" takes place there. In this case a transition zone between the swirling boundary layer flow and the main fully developed turbulent flow is absent.

With a generalized Reynolds analogy employed and under moderate values of the Prandtl number, the expression for the thermal resistance of completely turbulent flow was obtained in Reynolds [3]

$$
\frac{\lambda_{\mathrm{TPT}}}{8 \mathrm{St}}=\operatorname{Pr}_{t}+\frac{\tau_{\text {wall }} c_{p}}{U_{f}} R_{m},
$$

where $\lambda_{\text {TPT }}$ is the drag coefficient for water flow in TPTs; St is the Stanton number; $\tau_{\text {wall }}$ stands for TPT wall friction force; $c_{p}$ is isobaric specific heat; $U_{f}$ is the fluid velocity. The aim is to define the value of $R_{m}$ characterizing the heat transfer rate in TPT wall layer area.

A scheme of the wall flow in a TPT presents fig. 2. The space between the two adjacent protrusions (turbulators), the distance between them being equal 


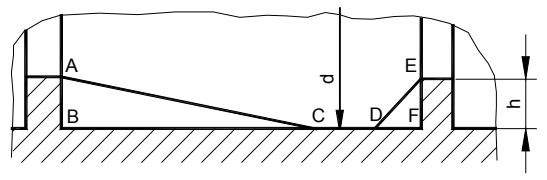

Figure 2: Wall flow in a TPT for heat transfer simulation. $A B C, D E F-$ vortex zone I; $C D$ - zone of relatively smooth helical flow II.

to $s$, is occupied both by the vortex zone I (ABC and DEF), whose total length along the wall is equal to $9 h$, and by the zone of a relatively smooth helical flow II (CD). The vortex quasi-stationary zone is practically insensitive to the protrusion orientation in the fluid flow over a wide range of incidence angles. It is assumed that $s>9 h$ and the thickness (width) of the protrusions (turbulators) is too small to be taken into account. The heat flux from the wall to the fluid flow in a TPT is defined as the sum of heat fluxes in zones I and II:

$$
q=q_{\mathrm{I}}+q_{\mathrm{II}}
$$

where $q_{\mathrm{I}}$ is the heat flux through the vortex area; $q_{\mathrm{II}}$ is the heat flux through the relatively smooth helical flow zone.

According to Reynolds [3] the vortex flow velocity in zone I is comparable to the dynamic velocity $v^{*}=\sqrt{\tau_{\text {wall }} / \rho}$, where $\rho$ stands for flow density. It is also assumed, that up to the moment of the vortex separation the heat is transferred to circulation flow by diffusion (i.e. by molecular transfer) near the wall. A distance of diffusion propagation over the circulation time $t \sim h / v^{*}$ is determined by the expression $Y \sim \sqrt{a t} \sim \sqrt{\frac{a h}{v^{*}}}$, where $a$ is the temperature conductivity coefficient. Then the value of heat flux in the vortex zone I per unit of area and per unit of time can be defined as

$$
q_{\mathrm{I}}=\frac{1}{t}\left[\rho c_{p}\left(T_{\text {wall }}-T_{s}\right)\right] Y=\rho c_{p}\left(T_{\text {wall }}-T_{s}\right) \sqrt{\frac{a}{t}}=\rho c_{p}\left(T_{\text {wall }}-T_{s}\right) \sqrt{\frac{a v^{*}}{h}},
$$

where $T_{\text {wall }}$ is the wall temperature and $T_{s}$ is the flow temperature at the outer boundary with the turbulent flow core.

The value of heat flux in the smooth flow zone could be expressed as

$$
q_{\mathrm{II}}=\frac{\lambda_{\text {wall }}}{Y_{h}}\left(T_{\text {wall }}-T_{s}\right),
$$

where $\lambda_{\text {wall }}$ is the heat conductance; $Y_{h}$ is the heat boundary layer thickness in smooth flow zone. Introducing the tube wall friction $\tau_{\text {wall }}=\frac{\mu v^{*}}{Y_{m}}\left(\mathrm{Y}_{m}\right.$ is the 
viscous sublayer thickness and $\mu$ is the dynamic viscosity coefficient) and assuming that $\mathrm{Y}_{m} \sim \mathrm{Y}_{h}$ Reynolds [3], the following expression is written as

$$
q_{\mathrm{II}}=\frac{\lambda_{\text {wall }} \tau_{\text {wall }}}{\mu v^{*}}\left(T_{\text {wall }}-T_{s}\right) .
$$

The flow temperature $T_{s}$ at the outer boundary with the turbulent flow core is supposed to be equal to the similar temperature for the vortex zone. The molecular heat transfer thermal resistance $R_{m}$ is defined near the TPT wall and is considered to be equal to the sum of the thermal resistances for both vortex and smooth flow zones taking into account their areas

$$
R_{m}=\frac{T_{\text {wall }}-T_{s}}{q_{\mathrm{I}} F_{\text {vort }}+q_{\mathrm{II}} F_{s m}},
$$

here $q_{\mathrm{I}}, q_{\mathrm{II}}$ are the specific heat fluxes through zones $\mathrm{I}$ and II; $F_{\text {vort }}=9 h \sqrt{(z s)^{2}+\left(\pi d_{i n}\right)^{2}}$ is the vortex zone area; $F_{s m}=\pi d_{i n}(z s)-F_{\text {vort }}$ is the smooth flow zone area, $d_{i n}$ is the tube inner diameter.

After substituting eqn (7) into eqn (2) the expression is got of $\mathrm{Nu}$ number ratio (in comparison to a plain tube) for water flow in TPTs, see Brodov et al. [4],

$$
\frac{\mathrm{Nu}_{\mathrm{TPT}}}{\mathrm{Nu}_{p l}}=\frac{\operatorname{Re} \operatorname{Pr} \lambda_{\mathrm{TPT}}}{8 \mathrm{Nu}_{p l}}\left\{\operatorname{Pr}_{t}+\frac{\operatorname{Re} \operatorname{Pr} \lambda_{\mathrm{TPT}} / 8}{B \sqrt{\operatorname{Re} \operatorname{Pr} \sqrt{\lambda_{\mathrm{TPT}} / 8}}+\frac{\operatorname{Re} \operatorname{Pr} \lambda_{\mathrm{TPT}} / 8}{[1-B] \operatorname{Re} \sqrt{\lambda_{\mathrm{TPT}} / 8}}}\right\}^{-1},
$$

where $\mathrm{Nu}_{p l}=0,023 \cdot \operatorname{Re}^{0,8} \operatorname{Pr}^{0,43} ; \quad B=9 h \sqrt{1+\left(\pi d_{i n} / z s\right)^{2}} / \pi d_{\text {in }}$. This equation is valid for $s>9 h$.

Turbulent Prandtl number $\operatorname{Pr}_{t}$ is the function of Reynolds and Prandtl numbers. As the flow swirling occurs in TPTs and it affects the flow core, the $\operatorname{Pr}_{t}$ value also depends on the profile parameters:

$$
\operatorname{Pr}_{t}=\operatorname{Pr}_{t}\left(\operatorname{Re}, \operatorname{Pr}, h, s, z, d_{i n}\right)
$$

This function could be found using experimental data for water flow in TPTs along with the expression (9). 


\section{Experimental study of heat transfer for liquid flow in TPT}

Experimental research of heat transfer for liquid flow in TPT was carried out with the use of a test set made as a heat exchanger of double pipe type with the areas of thermal and hydrodynamic stabilization (fig. 3).

In the research set the water moved in two closed-loop contours: the external (heating) and internal (heated) ones. The water is supplied either by countercurrent or by parallel flow.

When carrying out research tests the following parameters were measured: water temperatures at the inlets and outlets of each contour, test tube wall temperature, water temperature inside the test tube, water flow rates for both external and internal contours, hydraulic resistance of the tested tubes. All the temperature measurements were taken by chromel-copel thermocouples. The tube wall temperature was measured by twelve thermocouples in three sections over the tube length and in four points over each section perimeter.

Instrumental mean-square uncertainty for water flow rate measurements did not exceed $0.5 \%$, for water temperature differences (from 10 to $15^{\circ} \mathrm{C}$ ) it changed from 1 to $2 \%$, for "water-tube wall" temperature difference - from 3 to $6 \%$. The maximum mean-square error of the heat transfer coefficient did not exceed $\pm 7 \%$.

During research data processing the value of $\mathrm{Nu}$ number was determined with the TPT inside diameter measured on its plain (unrolled) part. Water velocity varied from 0.5 to $3.5 \mathrm{~m} / \mathrm{s}$, which corresponded to Reynolds numbers from $\mathrm{Re}_{w}=10000$ to $\mathrm{Re}_{w}=140000$.

The results of the tests carried out for plain tubes confirmed well-known equations for these tubes with the mean error of $\pm 7 \%$ Reynolds [3].

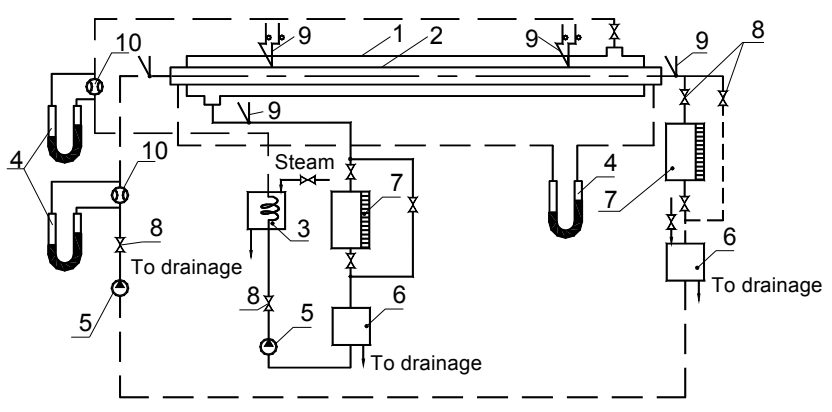

Figure 3: Experimental set for heat transfer and hydrodynamic research of water and air flow in TPTs. (1 - outer tube; 2 - test tube; 3 - steam-water heater; 4 - differential manometer; 5 - pump; 6 - storage tank; 7 - measuring tank; 8 - check valve; 9 - chromel-copel thermocouples; 10 - diaphragm.)

Fig. 4 shows the results of experimental data processed as a function of $\mathrm{Nu}$ number ratio versus $h / s^{*}$ parameter. One can see that there exists an optimal 
interval of $h / s^{*}$, where the maximal effect of heat transfer augmentation occurs. The experimental data for this area are generalized by the following expression:

$$
\frac{h}{s^{*}}=0.153 \mathrm{Re}_{w}^{-0.3},
$$

where $s^{*}=\sqrt{(z s)^{2}+\left(\pi d_{\text {in }}\right)^{2}}$ is the length of the TPT profile helical line.

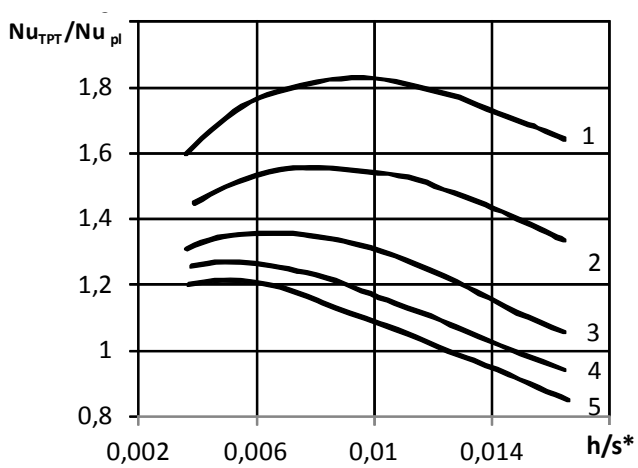

Figure 4: Function of heat transfer in a TPT versus tube profile geometrical parameters and the Reynolds number. $\left(1-\mathrm{Re}_{w}=10 \cdot 10^{3}\right.$; $2-20 \cdot 10^{3} ; 3-40 \cdot 10^{3} ; 4-60 \cdot 10^{3} ; 5-80 \cdot 10^{3}$.)

Fig. 5 presents the results of the comparative heat transfer research for air and water flow in a TPT. For water flow the $\mathrm{Nu}$ number ratio for the TPT and the plain tube decreases as Re number grows, and under certain tube profile parameters the TPT heat transfer coefficient becomes lower than that of the corresponding plain tubes. For air flow the $\mathrm{Nu}$ number ratio in the investigated range of parameters does not depend on the Reynolds number and is affected by the TPT profile parameters only. For the air flow in a TPT swirling is rather insignificant (unlike for the water flow) because the air viscosity is much smaller than that of water. The heat transfer augmentation is caused by the vortices separation and the wall layer turbulisation which depends upon the protrusions height and their relative location. In addition, the viscous sublayer thickness in a TPT is substantially smaller for air flow than for water and comparable in value with the height of the protrusions (characteristic size of the artificial roughness).

Experimental data on the $\mathrm{Nu}$ number ratio for water flow in TPTs with threestart knurling were approximated with the accuracy $\pm 10 \%$ by the following function

$$
\frac{\mathrm{Nu}_{\mathrm{TPT}}}{\mathrm{Nu}_{p l}}=\left\{1+338.6 \frac{h}{s}\left(1-\frac{2 h}{d_{i n}}\right)-1863.2\left[\frac{h}{s}\left(1-\frac{2 h}{d_{\text {in }}}\right)\right]^{2}\right\} \operatorname{Re}_{w}^{-0.43\left(\frac{h}{s^{*}}\right) 0.142},
$$

which is valid for $\mathrm{Re}_{w}$ numbers from 10000 to 240000 . 


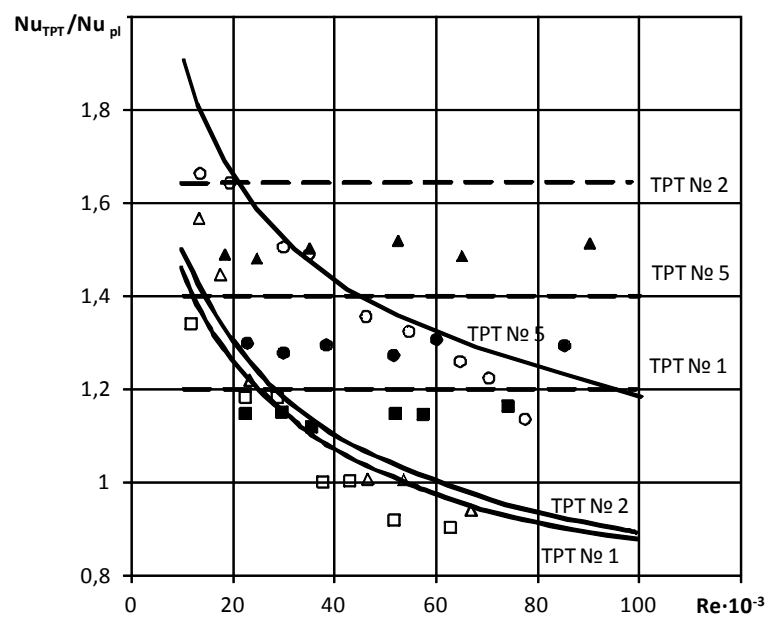

Figure 5: Comparison of heat transfer for water and air flows in a TPT. (- - water; $\square$ - TPT № $1: \mathrm{h} / \mathrm{s} / \mathrm{d}_{\text {in }}=0.8 / 7.0 / 17.0 \mathrm{~mm} ; \triangle-$ TPT № 2 - 1.1/15.3/17.0; ○ - TPТ № 5 - 1.0/48.0/17.0; - - - air;

- - TPТ № 1 - 0,8/7.0/17.0; $\boldsymbol{\Delta}$ - ТРТ № 2 - 1.1/15.3/17.0; •- ТРТ № 5 - 1.0/48.0/17.0.)

The experimental data were used to define the form of the function for the turbulent Prandtl number $\left(\operatorname{Pr}_{t}\right)$ from the semi-empirical expression (9) obtained in the simulation of the fluid hydrodynamics and heat transfer in a TPT. The final explicit form for the turbulent Prandtl number function $\operatorname{Pr}_{t}$ is

$$
\operatorname{Pr}_{t}=A \operatorname{Re}^{m} \operatorname{Pr}^{n}
$$

where $A=0.20, m$ varies from $0.163 \cdot \frac{h}{s^{*}}$ to $4.2 \frac{h}{s^{*}} ; n=0.52$.

Comparison of the experimental data on a number of TPTs to the results calculated by the expression (8) shows their adequate agreement, the error being less than $10 \%$. We believe that the proposed semi-empirical model makes it possible to calculate the heat transfer for water flow in twisted profile tubes.

Fig. 6 shows the results of hydraulic resistance research for water flow in TPTs. Data processing revealed that the TPT hydraulic resistance coefficient $\lambda_{\text {TPT }}$ depended only on $h / s$ ratio.

The experimental data on fig. 6 are described by the relationship

$$
\lambda_{\mathrm{TPT}}=\lambda_{\mathrm{pl}}\left[1+13\left(\frac{h}{s}\right)+94\left(\frac{h}{s}\right)^{2}\right]
$$

which is valid for $\operatorname{Re}_{w}$ from $10^{4}$ to $33 \cdot 10^{4} ; d_{i n}$ - from 14 to $33 \mathrm{~mm} ; h$ - from $0.01 \cdot d_{\text {in }}$ to $0.12 \cdot d_{i n} ; \mathrm{s}-$ from $9 h$ to $140 h ; z=1,2,3$. 


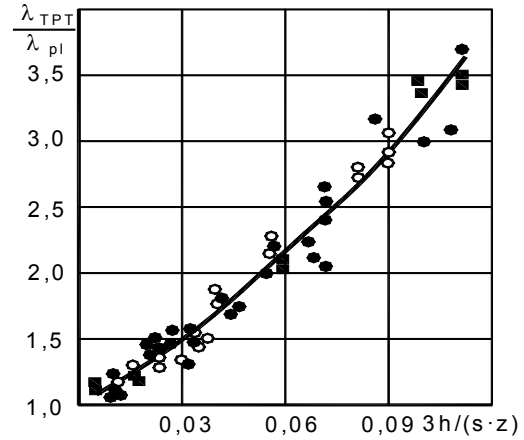

Figure 6: Generalized hydraulic resistance data for single phase flow in TPTs. ( - experimental data of Bogolyubov et al. [5]; ○ - data of Bogolyubov et al. [6]; • - authors' data; - calculation by eqn (13).)

\section{Oil flow in TPT bundles}

The research on hydrodynamics and heat exchange for the transverse flow of turbine oil through the tube bundles was carried out on a specially constructed experimental test set equipped with a cooled working tube, using a local thermal modeling method (fig. 7).

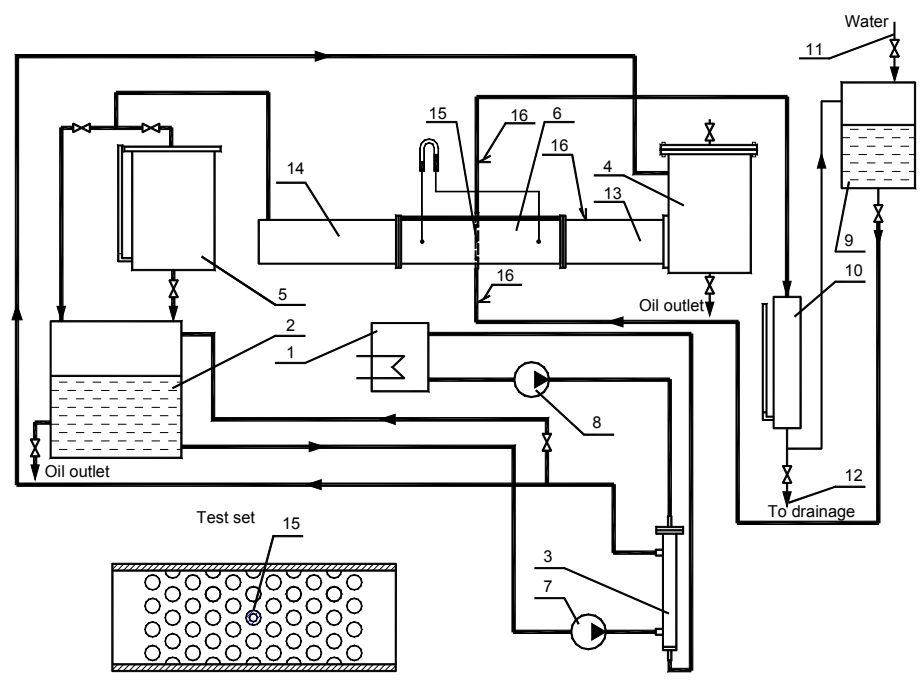

Figure 7: Experimental test set for oil flow research in tube bundles. (1 - thermostat; 2 - oil tank; 3 - heat exchanger; 4 - receiver; 5 - oil measuring tank; 6 - test module; 7 - oil pump; 8 - water pump; 9 - equalizing tank for cooling water; 10 - cooling water measuring tank; $11-$ cooling water supply; 12 - cooling water drain; 13 - stabilization section for the oil flow; 14 - outlet section; 15 - test tube; 16 - thermocouples.) 
In this case the temperature of the incident fluid flow corresponds to the flow temperature in front of the tube bundle. The experimental test set consists of three separate contours. The first contour serves for oil heating, the second one for oil supply, the third one - for cooling water supply (Brodov et al. [7]).

The test tube wall temperature is measured by the 5 thermocouples fixed in the middle section of the tube. When TPT is used as a test tube, the thermocouples were fixed both on the protrusion and in the groove of the tube. In all the basic experiments the TPT measuring section was oriented so that a thermocouple on the TPT protrusion was located in the frontal point with reference to the incident oil flow.

The aim of the research was to evaluate not only the test tube profiling effect but also the adjacent tubes influence on the heat transfer. There were 10 runs of experiments conducted for different variants of the tube bundle layout of plain tubes and TPTs with different profiling parameters.

The experiments were conducted in a wider range of oil velocity and temperature values as compared to the values of these parameters in the lot production oil coolers for the turbines' capacity ranging from 6 to $800 \mathrm{MW}$. To minimize the number of experiments, the tests for each TPT installation variant were carried out with typical values of $\mathrm{Re}_{\text {oil }}$ implemented by different combinations within the selected range of oil velocities and temperatures.

The mean-square uncertainty for water flow rate measurements was $0.2 \%$, for water temperature differences $-3.2 \%$, for temperature difference between the oil and the tube wall $-0.5 \%$. The maximum mean-square uncertainties of the oil heat transfer coefficient and the tube bundle pressure drop did not exceed $\pm 3.7 \%$ and $\pm 4 \%$ correspondingly.

Experimental data for the heat transfer coefficient are presented in a dimensionless form. This permits to generalize the heat transfer data under different velocities and temperatures of the incident oil flow:

$$
\mathrm{Nu}_{\text {oil }}^{*}=\frac{\mathrm{Nu}_{\text {oil }}}{\operatorname{Pr}_{\text {oil }}^{0.36}\left(\operatorname{Pr}_{f} / \operatorname{Pr}_{\text {wall }}\right)_{\text {oil }}^{0.25}}=f\left(\operatorname{Re}_{\text {oil }}\right) \text {. }
$$

An outside diameter of the plain test tube was used to define the numbers of Nusselt and Reynolds for oil flow $\left(\mathrm{Nu}_{\text {oil }}, \mathrm{Re}_{\text {oil }}\right)$.

The experiments on the oil flow heat transfer in tube bundles revealed that the efficiency of heat transfer for TPTs is higher than that of the plain tubes. As an example, fig. 8 presents the experimental data on the oil flow heat transfer for TPT tube bundles with various profile parameters, and fig. 9 shows the function of $\mathrm{Nu}_{\mathrm{TPT}} / \mathrm{Nu}_{p l}$ ratio versus Reynolds number $\mathrm{Re}_{\text {oil }}$. The maximum effect occurs when the increase of the heat transfer reaches $17.5 \%$ for the TPT tube bundle with profile parameters $s=8 \mathrm{~mm}, h=0.8 \mathrm{~mm}$.

The oil flow heat transfer for the tested TPT bundles is described by the generalized expression

$$
\mathrm{Nu}_{\mathrm{TPT}}=\mathrm{Nu}_{p l}\left(0.995+0.410 \frac{h}{s}\right) \operatorname{Re}_{\text {oil }}^{0.019} .
$$


The expression is valid for the values of Reynolds number $\mathrm{Re}_{\text {oil }}$ from 100 to 750 , for the TPT groove depth $h$ from 0.5 to $0.8 \mathrm{~mm}$, the groove pitch $s$ from 8 to $10 \mathrm{~mm}$ and the number of starts $z=3$.

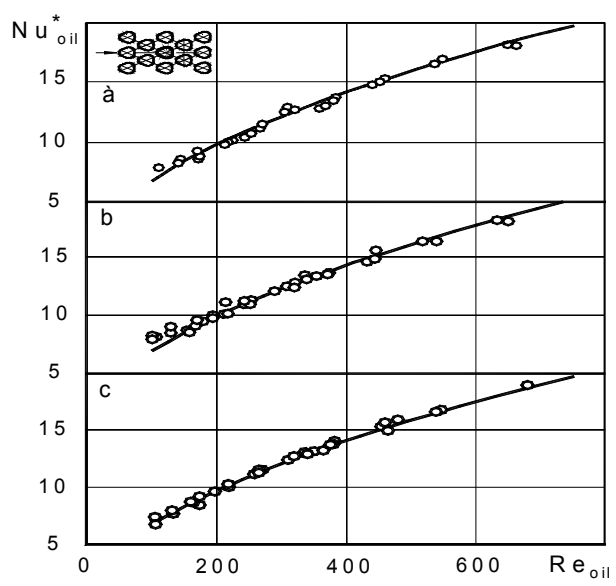

Figure 8: The results of the research on the heat transfer of the tested TPT in the TPT tube bundle. ( $\mathrm{O}$ - experimental data, _ experimental data approximation; a - TPT $(8 \times 0.5 \mathrm{~mm})$ (run 2), b - TPT $(8 \times 0.8 \mathrm{~mm})($ run 3$), \mathrm{c}-\mathrm{TPT}(10 \times 0.5 \mathrm{~mm})($ run 4$)$.)

The analysis of the research results of hydraulic resistance in oil cross flow obtained on the bundles of plain and profiled tubes showed that in the tested range of Reynolds numbers the tube profiling in tube bundles did not lead (within the experimental uncertainty) to a substantial change in the hydraulic resistance of TPT bundles in comparison to that of the plain tube bundles. The increase of the oil hydraulic resistance of the investigated TPT bundles could be compensated by an increase in the oil flow cross-section area, which is from 6.5 to $10.4 \%$ greater than in the plain tube bundle, depending on TPTs profile parameters.

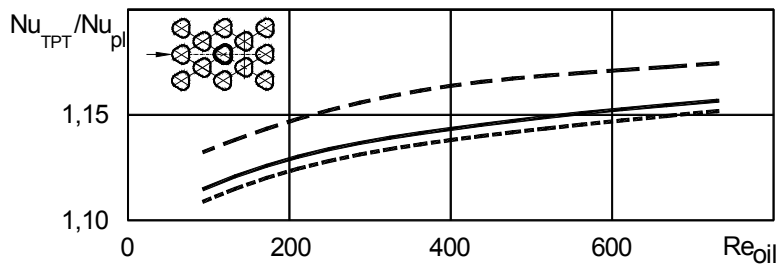

Figure 9: Heat transfer for TPT tube bundles in comparison to the plain tube bundle in the transverse oil flow. — run $2(8 \times 0.5 \mathrm{~mm}),--$ run $3(8 \times 0.8 \mathrm{~mm}),-----$ run $4(10 \times 0.5 \mathrm{~mm})$ 


\section{Conclusion}

The research conducted allows us to make the following conclusions:

1. The experiments showed the maximum increase in the heat transfer coefficient up to $80 \%$ for water flow through a TPT under the values of Reynolds number $\operatorname{Re}<20000$.

2. The heat transfer coefficient ratio $\left(\mathrm{Nu}_{\mathrm{TPT}} / \mathrm{Nu}_{p l}\right)$ decreases as Reynolds number increases for water flow in TPT; under some tube profile parameters and Re values the heat transfer coefficient ratio can be lower than 1 .

3. With the air passing through TPTs, the heat transfer coefficient ratio does not depend upon the Reynolds number, which is attributed to the different hydrodynamics in the TPT boundary layer for liquid and gas (i.e. a different value of the Prandtl number).

4. The increase of the hydraulic resistance for water flow through a TPT exceeds the increase of the heat transfer coefficient in the whole tested range of the process parameters, typical for power heat exchangers.

5. With the TPT oil cross-flow, the heat transfer coefficient increase ranges up to $15-17 \%$, while the TPT bundle hydraulic resistance does not virtually increase.

\section{References}

[1] Ryabchikov A.Yu., Aronson K.E., Brodov Yu.M., Khaet S.I., Blinkov S.N., Zhelonkin N.V. Modernization of heat exchangers in steam turbine units taking features of their operation at specific thermal power plants into account. Power technology and engineering. Thermal power stations. Vol. 44, № 3, S. 208-212, 2010.

[2] Aronson K.E., Brodov Yu.M., Mutovin A.T. Steam turbine heat exchangers modernization in view of operation features at various power stations. Lambert Academic Publishing: Nurnberg. 391 pp. 2011.

[3] Reynolds A.J. Turbulent Flows in Engineering Applications. Energija: Moscow, 408 pp. 1979.

[4] Brodov Yu.M., Gal'perin L.G., Chizhevskaya E.M. Calculation of heat transfer during water flow in profiled twisted pipes. Journal of Engineering Physics 49 (5), pp. 1254-1258, 1985.

[5] Bogolyubov Yu.N., Permyakov V.A., Grigoryev G.V. Hydraulic resistance of profile tubes with a spiral rolling. Energomashinostroenie; Moscow. № 12, pp. 19-21, 1976.

[6] Bogolyubov Yu.N. et al. Research of heat convection for single phase media flow in helically-profiled (twisted) tubes. NIIEinformenergomash: Moscow, № 1-77-16, pp. 12-15, 1977.

[7] Brodov Yu.M., Aronson K.E., Ryabchikov A.Yu., Lokalov G.A. An experimental investigation of heat transfer for viscous liquid flowing over bundles of smooth and profiled tubes as applied to turbine unit oil coolers. Thermal Engineering (English translation of Teploenergetika): Moscow, 55 (3), pp. 196-200, 2008. 